\title{
AN OPTIMIZATION FRAMEWORK FOR CLOUD-BASED DATA MANAGEMENT MODEL IN SMART GRID
}

\author{
P.M.Devie ${ }^{1}$, S.Kalyani ${ }^{2}$ \\ ${ }^{I}$ Assistant Professor, EEE Department, Kamaraj College of Engineering and Technology, Tamil Nadu, India \\ ${ }^{2}$ Professor\& Head, EEE Department, Kamaraj College of Engineering and Technology, Tamil Nadu, India
}

\begin{abstract}
Smart Grid $(S G)$ is an intelligent electricity network that incorporates advanced information, control and communication technologies to increase the reliability of existing power grid. With advanced communication and information technologies, smart grid deploys complex information management model. This paper presents a cloud service based information management model which opens the issues and benefits from the perspective of both smart grid domain and cloud domain of system model. The overall cost of data management includes storage, computation, upload, download and communication costs which need to be optimized. This paper provides an optimization framework for reducing the overall cost for data management and integration in smart grid model. In this paper, the optimization model focuses on optimizing the size of data items to be stored in the clouds under concern. The types of data items to be stored in the clouds are considered as customer behavior data and Phasor Measurement Units (PMU) data in the smart grid environment. The management model usually comprises of four domains viz., smart grid domain, cloud domain, broker domain and network domain. The present work focuses mainly on smart grid and cloud domain and optimization of cost related to these domains for simplicity of model considered. The proposed model is optimized using various evolutionary optimization techniques such as Genetic Algorithm (GA), Particle Swarm Optimization (PSO) and Differential Evolution (DE). The results of various techniques when implemented for proposed model are compared in terms of performance measures and a most suitable technique is identified for cloud based data management.
\end{abstract}

\section{Keywords: Smart grid, Information management, Optimization, Cloud Computing.}

\section{INTRODUCTION}

Smart grid is the replacement of aging power system by intelligent power system incorporating Energy Technology (ET) \& Information and Communication Technology (ICT). The implementation of smart grid technologies significantly increase the complexity of handling data in information management model, which means the overall cost of data manipulation in the data management model increases [2]. Cloud based data integration offers higher level of scalability, efficient data sharing mechanism, easy data handling for highly complex systems and better outsourcing for new entities. Hence, a design of efficient model and selection of suitable simulation tool is required for overall cost reduction of data management.

A next-generation distributed computing paradigm termed as cloud-computing technology plays a key role in information management by serving large data centers using cloud providers with massive storage and computation services [10]. Public utility services accessed by cloud consumers include resources such as network bandwidth, storage, processing power and cost, etc. Virtualization technology can provide resources to consumers based on their specified requirements and operating systems along with their applications is termed as virtual machine. Chaisiri et al. [6] proposed an optimal cloud resource provisioning algorithm to provision resources offered by multiple cloud providers. Endo et al. [3] discussed the main challenges which are inherent in the process of resource allocation for distributed clouds of high complex data management system. Adam Hahn et al. [4] proposed a modeling of secure testbed for smart grid networks, design of architecture for testbed and evaluation of performance applications to enhance the system reliability. Feng Guo et al. [5] developed a platform for real-time simulation of smart grid network and provide an effective approach to examine communication and distributed control related issues inn smart grids. The developed model can also be used to evaluate reconfiguration strategies of communication networks in smart grid environment. Fang et al. [7] discussed the evolving opportunities, designing of working models and applications of smart grid data management using cloud computing. Recently, researchers focus on key issues for handling the high complex data of large systems in smart grid system using cloud based services such as dynamic resource allocation, distributed computing, virtualization technology and data sharing mechanism, etc.,

The optimization of smart grid model comprising of minimizing the data storage and computation cost deals with equality constraints such as data redundancy and task execution constraints. Furthermore, the model can also be reformulated to incorporate other inequality constraints viz., data splitting, data exclusive, data upload and inter-cloud data transfer constraints for better understandability but it greatly increases the complexity of the model. This paper provides an optimization framework for reducing the overall cost of data management and integration in smart grid 
model. The proposed model is optimized using various evolutionary optimization techniques such as Real Coded Genetic Algorithm (RCGA), Particle Swarm Optimization (PSO) and Differential Evolution (DE). The results of various techniques when implemented for proposed model are compared in terms of performance measures and a most appropriate \& suitable technique is identified for efficient cloud based data integration.

\section{Nomenclature}

\begin{tabular}{|c|c|}
\hline $\mathrm{OC}$ & Overall Cost \\
\hline $\mathrm{Di}$ & Set of Data Items \\
\hline $\mathrm{U}$ & Set of Users \\
\hline $\mathrm{Tk}$ & Set of Tasks \\
\hline $\mathrm{C}$ & Set of Clouds \\
\hline $\mathrm{Di}^{\mathrm{S}}$ & Set of Data Items needs to be splitted \\
\hline $\mathrm{Di}^{\mathrm{O}}$ & $\begin{array}{l}\text { Set of Data Items needs to be stored } \\
\text { in one cloud }\end{array}$ \\
\hline $\mathrm{T}^{\mathrm{Di}}(\mathrm{d})$ & $\begin{array}{l}\text { Set of Tasks by considering data item } \\
\text { 'd' as input }\end{array}$ \\
\hline $\mathrm{T}^{\mathrm{Tk}}(\mathrm{t})$ & $\begin{array}{l}\text { Set of Tasks by considering the } \\
\text { output of task ' } t \text { ' as input }\end{array}$ \\
\hline $\mathrm{S}^{\mathrm{Di}}(\mathrm{d})$ & Size of Data Item 'd' \\
\hline $\mathrm{S}^{\mathrm{Tk}}(\mathrm{t})$ & Size of Output task ' $t$ ' \\
\hline $\mathrm{S}^{\mathrm{Di}}(\mathrm{d}, \mathrm{c})$ & $\begin{array}{l}\text { Size of portion of Data Item 'd' } \\
\text { stored in cloud 'c' }\end{array}$ \\
\hline $\mathrm{U}^{\mathrm{Tk}}(\mathrm{t})$ & $\begin{array}{l}\text { Set of Users requesting the output of } \\
\text { task ' } t \text { ' }\end{array}$ \\
\hline $\mathrm{P}^{\mathrm{UP}}(\mathrm{d}, \mathrm{c})$ & $\begin{array}{l}\text { Unit Price of uploading data item 'd' } \\
\text { to cloud 'c' }\end{array}$ \\
\hline
\end{tabular}

\begin{tabular}{|c|c|}
\hline $\mathrm{P}^{\mathrm{DW}}(\mathrm{c}, \mathrm{u})$ & $\begin{array}{l}\text { Unit Price of downloading data from } \\
\text { cloud 'c' to user ' } u \text { ' }\end{array}$ \\
\hline $\mathrm{P}^{\mathrm{S}}(\mathrm{c})$ & Unit Storage Price in cloud 'c' \\
\hline $\mathrm{P}^{\mathrm{ICT}}(\mathrm{c} 1, \mathrm{c} 2)$ & $\begin{array}{l}\text { Unit Inter-Cloud Transfer Price from } \\
\text { cloud 'c1' to cloud 'c2' }\end{array}$ \\
\hline $\mathrm{Cp}(\mathrm{t}, \mathrm{c})$ & $\begin{array}{l}\text { Computation cost charged by cloud } \\
\text { ' } c \text { ' for task ' } t \text { ' }\end{array}$ \\
\hline $\mathrm{B}^{\mathrm{UP}}(\mathrm{d}, \mathrm{c})$ & $\begin{array}{l}\text { Binary Variable to indicate whether } \\
\text { data item ' } \mathrm{d} \text { ' is uploaded to cloud ' } \mathrm{c} \text { ' } \\
\text { or not for data manipulation }\end{array}$ \\
\hline $\mathrm{B}^{\mathrm{Tk}}(\mathrm{t}, \mathrm{c})$ & $\begin{array}{l}\text { Variable to indicate whether task ' } t \text { ' } \\
\text { is performed in cloud ' } c \text { ' }\end{array}$ \\
\hline $\begin{array}{l}\mathrm{B}^{\mathrm{ICT}}(\mathrm{t} 1, \mathrm{t} 2, \mathrm{c} 1 \\
\mathrm{c} 2)\end{array}$ & $\begin{array}{l}\text { Binary Variable to indicate whether } \\
\text { intermediate data is transferring from } \\
\text { cloud 'c1' to 'c2' as result of task ' } \mathrm{t} 2 \text { ' } \\
\text { takes input from output of task ' } \mathrm{t} 1 \text { ' }\end{array}$ \\
\hline$\theta(d)$ & $\begin{array}{l}\text { Storage Splitting ratio of data item } \\
\text { ' } d \text { ' }\end{array}$ \\
\hline$\rho(d)$ & $\begin{array}{l}\text { Storage Redundancy ratio of data } \\
\text { item 'd' }\end{array}$ \\
\hline
\end{tabular}

\section{OVERVIEW OF SYSTEM MODEL}

Basically the system model comprises of four domainsSmart Grid domain, cloud domain, network domain and broker domain [1]. Our model focuses on smart grid and cloud domain and optimizes the control variables (data items) related to these domains only. Fig. 1 shows the cloud based data management model under concern for our study and depicts the networks involved in detail.

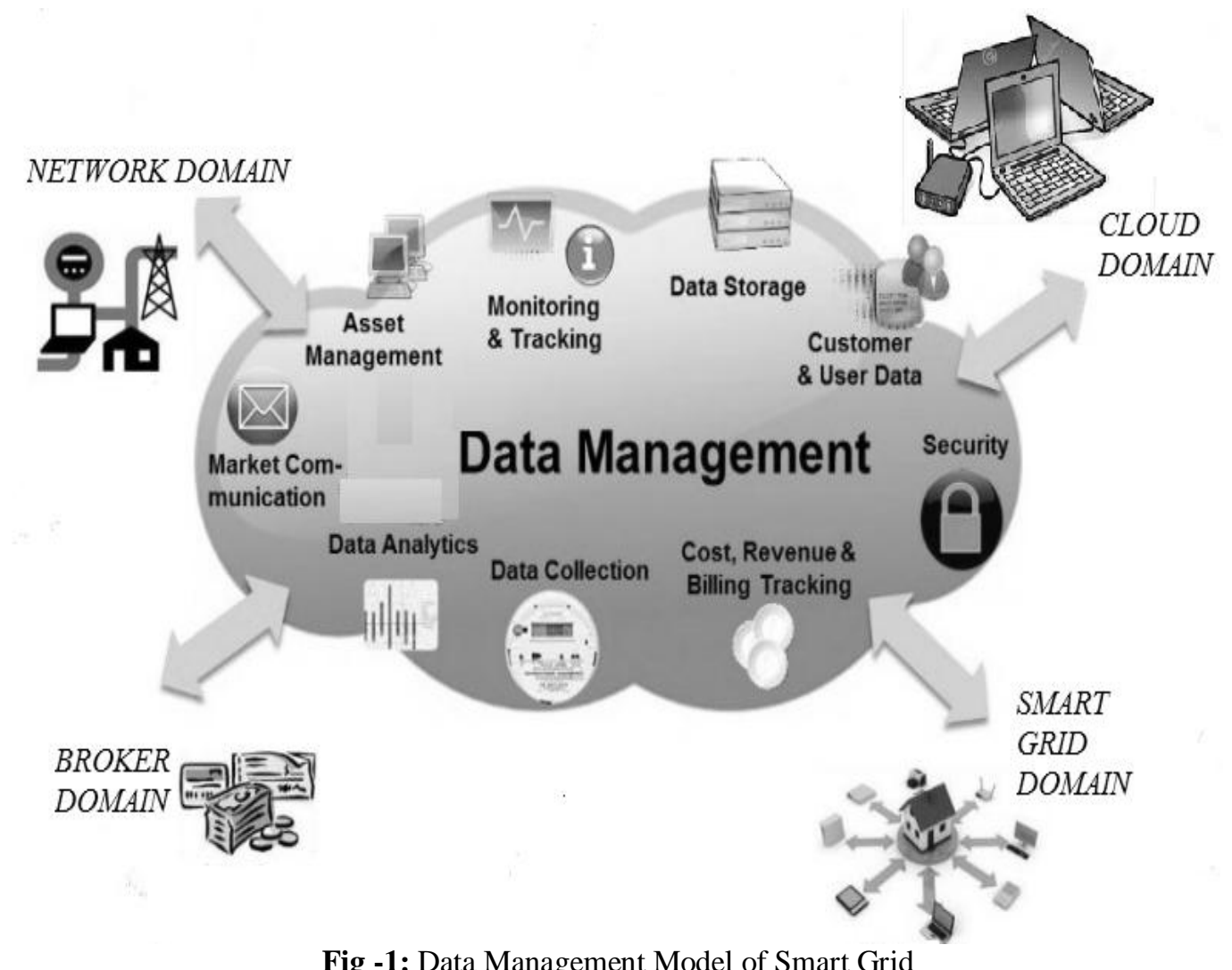

Fig -1: Data Management Model of Smart Grid 
1) Smart Grid Domain:

It is composed of seven sub domains as defined by the National Institute of Standards and Technology [21]. In this paper, we introduce three concepts related to data management - data item, computational project and user.

$>$ Data Item: It is a piece of information or object generated by information sources in the smart grid network such as Phasor Measurement Units [9], which should be stored securely. Data items are utilized by computational project for performing computation.

$>$ Computational Project: It consists of one or more tasks in which it uses some data items as input and performs required computing operations.

> User: A user is a consumer who is interested in getting some stored data items for access of usage.

2) Cloud Domain:

It consists of one or more clouds to provide storage and computation services for data items of smart grid network. Each cloud has its own pricing policy such as on-demand, reserve and spot pricing based on their requirement. For example, it is considered that a simple Amazon Storage Service is available in seven regions with different pricing policies [17].

3) Network Domain:

In this domain, networking providers own the network infrastructure \& communications and provide data transmission service between any two domains of smart grid network.

\section{4) Broker Domain:}

It consists of one or more brokers who act as a mediator between the smart grid and cloud domain for gathering information from cloud consumers and assisting the cloud providers in providing cloud services. The need for cloud brokers becomes inevitable due to increasing difficulty in satisfying the cloud consumer requirements [8]. Cloud brokers differ from traditional brokers and aim specifically to solve the smart grid data management problems.

In our model, the smart grid, network and cloud domain are of great concern. However, while programming the data of broker domain, the concerned model is updated at frequent intervals of time in existing smart grid network. To facilitate an efficient data integration of smart grid, our work focuses on optimization of cost of resource allocation (data item) for data storage and computation. The objective function of model combines the two sub models of smart grid viz., data storage and computation. For processing the data items collected from smart grid customers using data management model, we consider two Public Clouds (PC I and PC II) and one private cloud with pricing scheme of West-Northern California, US [17].

\section{AN OPTIMIZATION MODEL FOR DATA MANAGEMENT IN SMART GRID}

The overall cost minimization problem for cloud-based data storage and computation is formulated as follows:

\section{Objective Function:}

$$
\begin{aligned}
& \text { Min (Overall Cost) OC= } \\
& \sum_{d \in D i} \sum_{c \in C^{D i}(d)} S^{D i}(d, c) P^{S}(c) \\
& +\sum_{d \in D i c \in C^{D i}(d)} \sum^{D i}(d, c) P^{U P}(d, c) \\
& +\sum_{d \in D i c \in C^{D i}(d)} \sum_{u \in U^{D i}(d)} S^{D i}(d, c) P^{D W}(c) \\
& +\sum_{d \in D i c \in C^{D i}(d)} B^{U P}(d, c) S^{D i}(d, c) P^{S}(c) \\
& +\sum_{d \in D i i_{c \in C}} \sum_{C^{T k}} B^{T k}(t, c) C p(t, c) \\
& +\sum_{d \in D i c \in C^{D i}(d)} \sum^{U P}(d, c) S^{D i}(d) P^{U P}(d, c) \\
& +\sum_{t 1 \in T k_{t} \in T^{T k}} \sum_{(t 1)} \sum_{c 1 \in C^{T k}} \sum_{(t 1)} B_{c 2 \in C^{T k}(t 2)}^{I C T}(t 1, t 2, c 1, c 2) S^{T k}(t 1) P^{I C T}(c 1, c 2) \\
& +\sum_{t \in T k} \sum_{c \in C^{T k}} \sum_{(t)} B_{u \in U^{T k}(t)}^{T k}(t, c) S^{T k}(t) P^{D W}(c, u)
\end{aligned}
$$

(1)

Subject to

* Equality Constraints

1. Data Redundancy Constraint

$$
\sum_{c \in C^{D i}(d)} S^{D i}(d, c)=\rho(d) S^{D i}(d), \forall d \in D i
$$

2. Task Execution Constraint

$$
\sum_{c \in C^{T k}(t)} B^{T k}(t, c)=1, \forall t \in T k
$$

\section{* Inequality Constraints}

3. Data Splitting Constraint

$$
S^{D i}(d, c) \leq \frac{\rho(d) S^{D i}(d)}{\theta(d)}, \forall c \in C^{D i}(d), \forall d \in D i^{S}
$$

4. Data Exclusive Constraint

$$
\begin{aligned}
& \frac{S^{D i}(d, c)}{\rho(d) S^{D i}(d)}=\{0,1\}, \forall c \in C^{D i}(d), \forall d \in D i^{O} \\
& S^{D i}(d, c) \in[0, \infty), \forall c \in C^{D i}(d), \forall d \in D i .
\end{aligned} ;
$$


5. Data Upload Constraint

$$
\begin{aligned}
& \frac{\sum_{t \in T k^{D i}} B^{T k}(t, c)}{\left|T k^{D i}(d)\right|} \leq B^{U P}(d, c) \\
& \leq \sum_{t \in T k^{D i}(d)} B^{T k}(t, c), \forall c \in C^{D i}(d), \forall d \in D i ;
\end{aligned}
$$

6. Inter Cloud Data Transfer Constraint

$$
\begin{aligned}
& \frac{1}{2}\left(B^{T k}(t 1, c 1)+B^{T k}(t 2, c 2)\right)-\frac{1}{2} \leq B^{I C T}(t 1, t 2, c 1, c 2) \\
& \leq \frac{1}{3}\left(B^{T k}(t 1, c 1)+B^{T k}(t 2, c 2)\right)+\frac{1}{3}, \forall c 1 \in C^{T k}(t 1), \\
& \forall c 2 \in C^{T k}(t 2), \forall t 2 \in T^{T k}(t 1), \forall t 1 \in T k ;
\end{aligned}
$$

In this model, the fitness function eqn. (1) is considered as which minimizes the overall cost of smart grid data management (data upload, data download, communication, inter cloud data transfer, transfer in and transfer out costs).

\section{Elucidation of Constraints:}

$\checkmark \quad$ Eqn. (2) represents the data redundancy constraint, which consist of storage redundancy ratio $(\rho(d))$ of data item which is assumed to be 2 . Total size of data items stored in cloud domain is the product of size of data item and its storage redundancy ratio.

$\checkmark \quad$ Eqn. (3) represents the task execution constraint i.e., each task is exactly executed in one of the clouds.

$\checkmark \quad$ Eqn. (4) represents the data splitting constraint, which comprise of storage splitting ratio $\theta(d)$ should not be less than the reciprocal of total size of data items stored in cloud.

$\checkmark \quad$ Eqn. (5) represents the data exclusive constraint, which requires the set of data items to be completely stored in one of the clouds.

$\checkmark \quad$ Eqn. (6) represents the data upload constraint, which ensures $\mathrm{B}^{\mathrm{UP}}(\mathrm{d}, \mathrm{c})$ to be ' 1 ' only for some task with $\mathrm{B}^{\mathrm{Tk}}(\mathrm{t}, \mathrm{c})$ is one.

Eqn. (7) represents the inter cloud data transfer constraint which ensures $\mathrm{B}^{\mathrm{ICT}}(\mathrm{t} 1, \mathrm{t} 2, \mathrm{c} 1, \mathrm{c} 2)$ to be ' 1 ' if and only if $B^{\mathrm{Tk}}(\mathrm{t} 1, \mathrm{c} 1)=\mathrm{B}^{\mathrm{Tk}}(\mathrm{t} 2, \mathrm{c} 2)=1$.i.e., If task ' $\mathrm{t} 1$ ' is executed in cloud ' $\mathrm{c} 1$ ', task ' $\mathrm{t} 2$ ' is executed in cloud ' $\mathrm{c} 2$ ' and ' $\mathrm{t} 2$ ' takes the output of ' $\mathrm{t} 1$ ' as input, then $\mathrm{c} 1$ will transfer the output of ' $\mathrm{t} 1$ ' to ' $\mathrm{c} 2$ '.

In this paper, the Optimized cost of data management is evaluated by incorporating the equality constraints in the fitness function. The use of inequality constraints given by Eqns. (4) - (7) increases the complexity of proposed model. However, the inclusion of inequality constraints in the optimization algorithm does not create significant impact in the solution variables. Hence for simplicity of mathematical analysis, the inequality constraints are neglected in the present work.

\section{OPTIMIZATION TECHNIQUES USED}

In this paper, the proposed optimization model is evaluated using various techniques such as Real Coded Genetic Algorithm (RCGA), Particle Swarm Optimization (PSO) and Differential Evolution (DE) for minimizing the overall cost of data integration (Eqn. 1) in smart grid network.

\subsection{Real Coded Genetic Algorithm:}

Genetic Algorithm (GA) belongs to the class of randomized heuristic search techniques. GA is a general purpose search procedure that uses the principles inspired by natural genetic populations to evolve solution [16]. The traditional GA uses binary representation of strings which is not preferred in continuous search space domain. Real Coded Genetic algorithm (RCGA) gives a straightforward representation of chromosomes by directly coding all variables. The chromosome $X$ is represented as $X=\left\{S^{D i}, S^{T k}\right\}$, where $S^{D i}$ and $S^{\mathrm{Tk}}$ denotes the size of data items stored in every cloud for storage and computation respectively.

Unlike traditional binary coded GA, decision variables can be directly used to compute the fitness value. The RCGA uses selection, crossover and mutation operators to reproduce offspring for the existing population [12]. The RCGA-Smart grid model incorporates Roulette Wheel selection to decide chromosomes for next generation. The selected chromosomes are placed in a matting pool for crossover and mutation operations. The crossover operation enhances the global search property of GA and mutation operation prevents the permanent loss of any gene value. In this work, Arithmetic Crossover and Polynomial Mutation, described in Ref. [13], has been used to perform crossover and mutation respectively. The detailed procedure of RCGA applied for the problem of SG data management is shown in the form of flowchart in Fig. 2.

\subsubsection{PSO Algorithm:}

Particle Swarm Optimization (PSO) is an evolutionary computation technique developed by Kennedy and Eberhart in 1995, inspired by social behavior of birds flocking in a multidimensional space. The system is initialized with a population of random solutions and searches for optima by updating the generations [19]. In PSO, each single solution is called as particle. All particles have fitness value, evaluated by fitness function to be optimized, and have velocities, which direct the flying of the particles [20]. To discover the optimal solution, each and every particle is updated by two 'best' values. The first one called Pbest is the best position particle has achieved so far. The second best value is the overall best value obtained among all particles in the population, called as Gbest. After finding these two best values, each particle changes its velocity and position according to the cognition part (Pbest) and social part (Gbest). The update equations for particle's velocity and position are given by Eqns. (8) and (9).

$$
\begin{aligned}
& V_{i d}^{k+1}=\omega^{*} V_{i d}^{k}+c_{1} * \operatorname{rand}_{1} *\left(\text { Pbest }_{i d}^{k}-X_{i d}^{k}\right) \\
& +c_{2} * \operatorname{rand}_{1} *\left(\text { Gbest }_{d}^{k}-X_{i d}^{k}\right)
\end{aligned}
$$




$$
\begin{aligned}
& X_{i d}^{k+1}=X_{i d}^{k}+V_{i d}^{k} \\
& \omega=\omega_{\max }-\frac{\omega_{\max }-\omega_{\min }}{\text { Max.Iterations }} * \text { Current.Iteration }
\end{aligned}
$$

Where $\mathrm{w}$ is the inertia weight calculated by Eqn. (10), $\mathrm{V}_{\text {id }}$ is the particle velocity, $X_{i d}$ is the current particle position (solution), rand $_{1}$ is a random number between $(0,1), \mathrm{c} 1$ and c2 indicates cognition and social learning factors respectively.

\section{PSO Algorithm for SG Data Management}

1) Randomly initialize a population of particles with position $\mathrm{X}_{\mathrm{id}}(\mathrm{C}, \mathrm{c})$ and velocities $\mathrm{V}_{\text {id }}$ of the $\mathrm{i}^{\text {th }}$ particle in $\mathrm{d}^{\text {th }}$ dimension.

2) Set the parameters of PSO, $\mathrm{C}_{1}=\mathrm{C}_{2}=2, \mathrm{~W}_{\max }=0.9$, $\mathrm{W}_{\text {min }}=0.5$.

3) Evaluate the fitness of each particle in the population for SG model built with each particle's position $\left(S^{D i}\right.$ and $S^{T k}$ ).

4) Compare the current position with particle's previous best experience, Pbest, in terms of fitness value and hence update Pbest for each particle in the population.

5) After updating the Pbest, choose the best value among all the particles in Pbest and call it as Global best, Gbest.

6) Update the particle's velocity using Eqn. (8) and clamp to its minimum $\left(\mathrm{V}_{\min }\right)$ and maximum $\left(\mathrm{V}_{\max }\right)$ limit, whichever violates.

7) Move to the next position of the particle using Eq. (9) bounded to its upper and lower limits.

8) Stop the algorithm and print the near optimal solution *(final Gbest) if termination criterion or maximum iterations are reached; otherwise loop to Step 3 and continue the process.

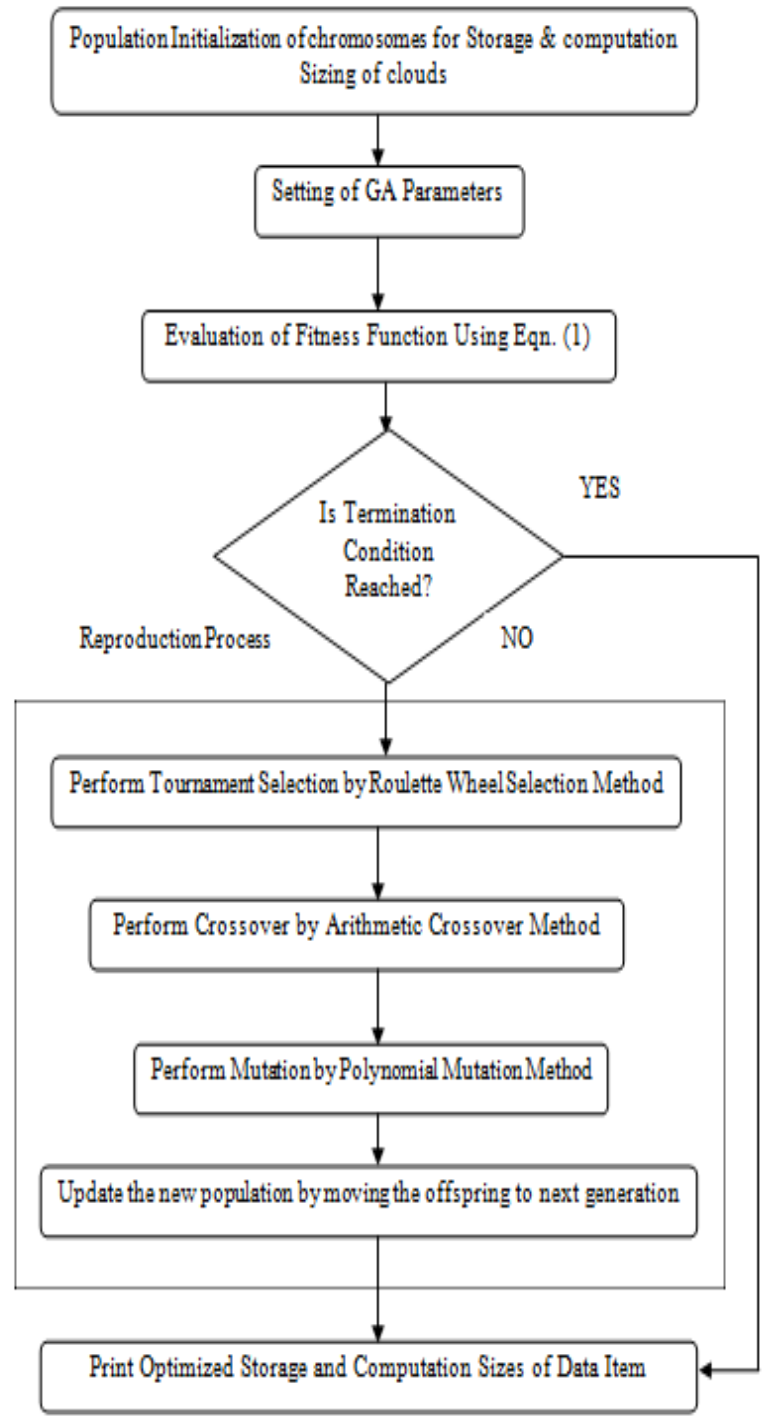

Fig -2: Flowchart for Real Coded Genetic Algorithm (RCGA)

\subsection{Differential Evolution Algorithm:}

Differential Evolution, one of the evolutionary optimization techniques, was introduced by Storn and Price in 1995 [14]. $\mathrm{DE}$ is highly effective and suitable for high dimensionality problems, which deals with high nonlinearity and multiple optima. Like any other evolutionary algorithm, DE also starts with random initialization of population vector with uniform distribution in search space. DE has three operations-mutation, crossover and selection [15].

The crucial idea behind DE is that crossover and mutation are used for generating trial vectors [18]. Mutation operation generates new parameter vector by adding the weighted difference between two population vectors to a third vector. Crossover operation mixes the mutated vector with target vector to yield a trial vector. Based on the computation of trial vectors, selection operation decides the survival of new vectors to the next generation. There are several strategies that can be used in DE algorithm. In this paper, we have used a commonly used strategy denoted as 'DE/rand/1/bin' 
[14]. In this representation, 'rand' indicates a random mutant vector to be chosen; ' 1 ' the number of difference vectors used and 'bin' denotes the crossover scheme. The DE algorithm used in this work for SG Data Management is briefed herein.

\section{DE Algorithm for SG Data Management}

1) Randomly initialize a population of individuals $X_{i d}$ denoting the ith individual in dimension.

2) Specify the DE parameters; difference vector scale factor $\mathrm{F}=0.05$, minimum and maximum crossover probability $\mathrm{CR}_{\min }=0.1$ and $\mathrm{CR}_{\max }=0.9$.

3) Evaluate the fitness value of each individual in the population. The fitness value is the overall cost of proposed data management of smart grid.

4) Generate mutant vector for each individual $x_{i}$ according to Eqn. (11)

$$
V_{i}=X_{S_{1}}+F *\left(X_{S_{2}}-X_{S_{3}}\right)
$$

The indices $s_{1}, s_{2}$ and $s_{3}$ are randomly chosen from population size. It is important to ensure that these indices are different from each other and also from the running index $\mathrm{i}$.

5) Perform crossover to yield the trial vector $u$ by combining the mutant vector $\mathrm{v}$ with target vector $\mathrm{x}$ using Eqn. (12).

$$
U_{i j}=\left\{\begin{array}{lll}
V_{i j} \operatorname{rand}(j) \leq C R & \text { or } & j=\operatorname{randn}(i) \\
X_{i j} \operatorname{rand}(j) \leq C R & \text { or } & j \neq \operatorname{randn}(i)
\end{array}\right.
$$

Where rand $(j) \in[0,1]$ is the $j^{\text {th }}$ evaluation of a uniform random generator number. randn $(i) \in\{1,2$, . $\ldots, D\}$ is a randomly chosen index ensuring that ui gets at least one element form mutant vector, vi. CR is the time varying crossover probability constant determined using (13).

$$
C R=C R_{\min }-\frac{C R_{\max }-C R_{\min }}{\text { Max.Iterations }} * \text { Iteration }
$$

6) Perform selection operation based on fitness value and generate new population. If the trial vector $u_{i}$ yields a better fitness, then $x_{i}$ is replaced by $u_{i}$, else $x i$ is retained at its old value.

7) If stopping criterion (maximum iterations) is reached, stop and print the optimized parameter set $\left(\mathrm{S}^{\mathrm{Di}}\right.$ and $\mathrm{S}^{\mathrm{Tk}}$ ); else increase iteration count and loop to Step 3.

\section{RESULTS \& DISCUSSIONS}

The program code for optimizing the cost of data management using various evolutionary algorithms were performed in MATLAB environment with processor of below specified configuration.

\section{Processor Configuration:}

The construction of SG Data integration model was performed in MATLAB environment using Matlab 8.1 version. The optimization framework is designed on PC with $2.5 \mathrm{GHz}$ core i3 CPU and 4 GB RAM.

The simulation is performed by considering one large electric utility, two public clouds (PC-I and PC-II), and two types of data item (customer behavior data and PMU data). This electric utility maintains single private clouds by itself. The number of residential areas served by this utility was varied from 50 to 500 with increment of 50 . The number of households in each residential area were set to $G(a, b, c, d)$, where ' $G$ ' denotes Gaussian variable with mean of ' $a$ ', variance of ' $b$ ', and range of $[c, d]$.

Each household is equipped with one smart meter. It is assumed that each smart meter generates one megabyte of information per day with sampling interval is 15 minutes. The data aggregated from the smart meters in each residential area is represented as one data item and categorized as the customer behavior data. These data items can be stored in public and/or private clouds. The sensitivity of the customer demand (behavior) data forces some of the data items to be stored only in the private clouds, and the storage splitting ratio and the redundancy ratio are both set to 2 .

It is further assumed that all the data items are stored for a period of six months and its cost of storage is evaluated in one day. The unit storage price for one gigabyte per month of PC-I is \$0.125 and that of PC-II is \$0.14. The unit storage price for one gigabyte per month of a private cloud is uniformly distributed between $\$ 0.15$ and $\$ 0.2$.

The unit upload (download) price is equal to the sum of the transfer-in (transfer-out) price charged by the clouds and the communication price charged by networking providers. The average transfer-in (transfer-out) price of PC-I and PC-II is $\$ 0$ (\$0.12) per gigabyte. The average transfer-in (transferout) price of the private clouds is assumed to be $\$ 0.2(\$ 0.2)$ per gigabyte. The communication price was assumed to be $\mathrm{G}(0.1,0.05,0.05,0.15)$.

Computational Project is evaluated which analyzes electricity saving products once a month (30 days). The settings of clouds, residential areas, upload prices, and download prices are similar to storage pricing of data item. In addition, the price of PC-I for high memory on-demand computational task is $\$ 2.28$ per hour, and that of PC-II is $\$ 2.00$ per hour. The price of private clouds for computational task is $\$ 3.00$ per hour. The unit inter-cloud transfer price between clouds $\mathrm{c} 1$ and $\mathrm{c} 2$ is equal to the sum of the transfer-out price of $\mathrm{c} 1$, the transfer-in price of $\mathrm{c} 2$, and the communication price between $\mathrm{c} 1$ and $\mathrm{c} 2$.

The proposed optimization model is framed under the above specified scenario and the optimized cost of data management is evaluated using different evolutionary algorithms such as Real Coded Genetic Algorithm, Particle 
Swarm Optimization and Differential Evolution and their detailed algorithms were already discussed in previous section. The performance of each evolutionary optimization technique when applied for the proposed data integration model is evaluated and depicted in Table 1. It is evident from Table 1 that consistency of each algorithm is measured in terms of statistical parameters namely mean and standard deviation. Further, the near global optimal solution of sizing of data items for storage \& computation and the total overall cost of storage (fitness function) obtained in various algorithms are compared in Table 1.

On comparing the results of various techniques, the Particle Swarm Optimization (PSO) technique is shown to produce a better performance in terms of minimum fitness function and convergence time per trial. Fig. 3 shows the convergence characteristics of different evolutionary algorithms. It is also seen from Fig.3 that PSO algorithm outperforms other evolutionary algorithms as it shows a quicker convergence (i.e., only for convergence) and also the value of fitness function is greatly reduced.

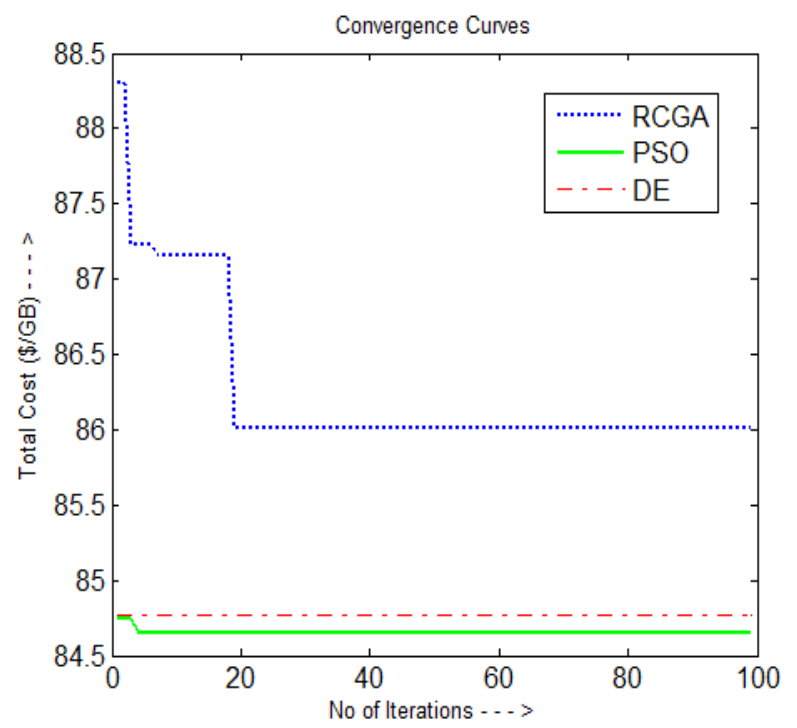

Fig -3: Convergence Curves for Different Optimization Techniques

Table -1: Performance Evaluation of Various Optimization Tool

\begin{tabular}{|c|c|c|c|c|c|c|c|c|c|c|}
\hline & \multirow{3}{*}{\begin{tabular}{|l|} 
Parameters \\
$\begin{array}{l}\text { Storage Size of } \\
\text { each cloud (GB) }\end{array}$ \\
\end{tabular}} & \multicolumn{9}{|c|}{ Optimization Technique Used } \\
\hline & & \multicolumn{3}{|l|}{ RCGA } & \multicolumn{3}{|l|}{ PSO } & \multicolumn{3}{|l|}{$D E$} \\
\hline & & 8.510 & 6.490 & 0.000 & 10.000 & 5.000 & 0.000 & 9.902 & 5.081 & 0.017 \\
\hline \multirow{6}{*}{ 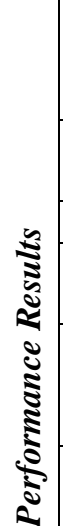 } & $\begin{array}{ll}\begin{array}{l}\text { Computation Size } \\
\text { of }\end{array} & \text { each } \\
\text { cloud(GB) } & \end{array}$ & 0.063 & 0.064 & 0.073 & 0.100 & 0.100 & 0.000 & 0.063 & 0.098 & 0.039 \\
\hline & $\begin{array}{ll}\text { Fitness } & \text { Function } \\
(\$ / G B) & \\
\end{array}$ & \multicolumn{3}{|c|}{84.7464} & \multicolumn{3}{|c|}{84.6531} & \multicolumn{3}{|c|}{84.6868} \\
\hline & Mean(\$/GB) & \multicolumn{3}{|c|}{85.9553} & \multicolumn{3}{|c|}{84.6534} & \multicolumn{3}{|c|}{84.8491} \\
\hline & $\begin{array}{l}\text { Standard } \\
\text { Deviation }\end{array}$ & \multicolumn{3}{|c|}{0.79074} & \multicolumn{3}{|c|}{0.0033834} & \multicolumn{3}{|c|}{0.10155} \\
\hline & \begin{tabular}{l}
\multicolumn{3}{l}{ Convergence } \\
Time per Trial \\
$(\mathrm{sec})$
\end{tabular} & \multicolumn{3}{|c|}{0.16365} & \multicolumn{3}{|c|}{0.05905} & \multicolumn{3}{|c|}{0.33835} \\
\hline & $\begin{array}{l}\text { No of Iteration } \\
\text { for Convergence }\end{array}$ & \multicolumn{3}{|l|}{33} & \multicolumn{3}{|l|}{4} & \multicolumn{3}{|l|}{1} \\
\hline
\end{tabular}

\section{CONCLUSION}

The proposed work presents an optimization model for cloud-based data management of smart grid network. The proposed model deals with optimization framework that incorporates the data items such as customer behavior data and PMU data of smart grid network. This work also aims to devise an appropriate evolutionary technique for overall cost minimization of data integration. It was proven that PSO algorithm outperforms other evolutionary algorithms by analyzing the simulation results of various evolutionary algorithms and hence is identified as a suitable optimization tool. In this work, PSO simulation for proposed model takes comparatively lesser time which makes it better to implement for online computation of smart grid network. The model can be extended further to include the detailed data pattern of smart grid such as customer account data and electric vehicle charging data in deregulated environment.

\section{REFERENCES}

[1] Xi Fang, Dejun Yang and Guoliang Xue "Evolving Smart Grid Information Management Cloudward: A Cloud Optimization Perspective", IEEE Transactions on Smart Grid, Vol.4, No.1, March 2013,pp. 111-119.

[2] Zhong Fan, Parag Kulkurani, et.al "Smart Grid Communications: Overview of Research Challenges, Solutions, and Standardization Activities", IEEE Communication Surveys \& Tutorials, Vol.15, No.1, 2013,pp. 21-38.

[3] P. Endo, A. de Almeida Palhares, N. Pereira, G. Goncalves, D. Sadok, J. Kelner, B. Melander, and J. Mangs. Resource allocation for distributed cloud: concepts and research challenges. IEEE Network, pages 42-46, 2011. 
[4] Adam Hahn, Aditya Ashok, Siddharth Sridhar and Manimaran Govindarasu "Cyber - Physical Security Testbeds: Architecture, Application, and Evaluation for Smart Grid", IEEE Transactions on Smart Grid, Vol.4, No.2, June 2013,pp. 847 -855.

[5] S. Chaisiri, B.-S. Lee, and D. Niyato, "Optimization of resource provisioning cost in cloud computing," IEEE Trans. Services Comput., vol.5, no. 2, pp. 164177, Apr.-Jun., 2012.

[6] X. Fang, S. Misra, G. Xue, and D. Yang, "Managing smart grid information in the cloud: Opportunities, model, and applications," IEEE Netw., vol. 26, no. 4, pp. 32-38, Jul.-Aug., 2012.

[7] S. Sakr, A. Liu, D.M. Batista, andM.Alomari, "Asurvey of large scale data management approaches in cloud environments," IEEE Commun. Surveys Tuts., vol. 13, no. 3, pp. 311-336, 2011.

[8] X. Fang, S. Misra, G. Xue, and D. Yang. Smart grid the new and improved power grid: A survey. IEEE Communications surveys and tutorial (Digital Object Identifier: 10.1109/SURV.2011.101911.00087).

[9] Simmhan, Y.; Aman, S.; Kumbhare, A.; Rongyang Liu; Stevens, S.; Qunzhi Zhou; Prasanna, V., "CloudBased Software Platform for Big Data Analytics in Smart Grids," Computing in Science \& Engineering, vol.15, no.4, pp.38,47, July-Aug. 2013.

[10] Wu C, Tzeng G, Goo Y, Fang W. "A real-valued genetic algorithm to optimize the parameters of support vector machine for predicting bankruptcy", Expert System Applications 2007; 32(2):397-408.

[11] Goldberg D. "Genetic algorithms in search optimization and machine learning" Addison-Wesley; 1989.

[12] Deb K. "Multi-objective optimization using evolutionary algorithms" Wiley; 2001.

[13] Storn R, Price K." Differential evolution - a simple and efficient heuristic for global optimization over continuous spaces" Journal on Global Optimization: 11(4), pp. 341-359, 1997.

[14] Arya L, Choube S, Arya R. "Differential evolution applied for reliability optimization of radial distribution systems", International Journal of Electric Power and Energy Systems, Volume 33, Issue 2, pp. 271-277,2011.

[15] Kalyani S and Swarup K S, "Pattern Analysis and Classification for Security Evaluation in Power Networks", International Journal of Electric Power and Energy Systems, volume 44 ,pp. 547-560,2013.

[16] Amazon Simple Storage Service [Online]. Available: http://aws. amazon.com/s3/.

[17] Zhou S, Wu L, Yuan X, Tan W. "Parameters selection of SVM for function approximation based on differential evolution", Proceedings of the international conference on intelligent systems and knowledge engineering (ISKE 2007), Chengdu, China; 2007.

[18] Kennedy J, Eberhart R, et al. "Particle swarm optimization" Proceedings of IEEE international conference on neural networks, vol. 4. Piscataway, NJ: IEEE; pp. 1942-1948, 1995.
[19] Mostafa H, El-Sharkawy M, Emary A, Yassin K. "Design and allocation of power system stabilizers using the particle swarm optimization technique for an interconnected power system" International Journal of Electric Power and Energy Systems, Volume 34, Issue 1,pp. 57-65, January 2012.

[20] National Institute of Standards and Technology, NIST Framework and Roadmap for Smart Grid Interoperability Standards, Release 1.0, 2010.

\section{BIOGRAPHIES}

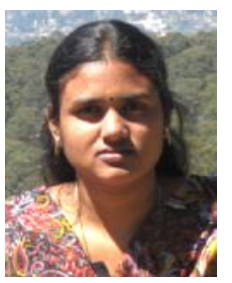

P. M. Devie received her bachelor's degree in Electrical and Electronics Engineering from KLN College of Information Technology, Madurai, in the year 2012. She completed her Masters Degree in Power Systems Engineering from Kamaraj College of Engineering \& Technology, Virudhunagar, in the year 2014. She is currently working as Assistant Professor in the Kamaraj College of Engineering \& Technology, Virudhunagar and pursuing Ph.D. in Anna University, Chennai under Part-Time. Her research interests are power system stability, data mining techniques for power system classification problems, smart grid technologies and its applications.

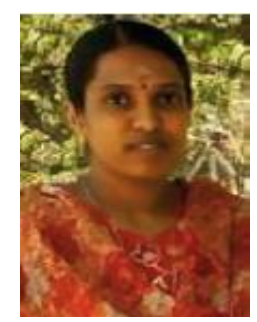

S. Kalyani is currently working as Professor and Head in the Department of Electrical \& Electronics Engineering at Kamaraj College of Engineering \& Technology, Virudhunagar. She obtained her PhD degree from Indian Institute of Technology Madras, Chennai in year 2011. She received her Master's Degree in Power Systems Engineering from Thiagarajar College of Engineering, Madurai in December 2002 and Bachelors Degree in Electrical and Electronics Engineering from Alagappa Chettiar College of Engineering Karaikudi, in the year 2000. She has about 12 years of teaching experience at various levels. Her research interests are power system stability, Pattern Recognition, Machine Learning, Soft Computing Techniques to Power System studies. She is a Member of IEEE, member of IE (India) and also life member of ISTE. 